\title{
Therapeutic Potential of Green Tea Catechins - A Magical Herb
}

\author{
G. Swarnalatha ${ }^{1 *}$, B. Surendra Nath ${ }^{1}$, N. Laxmana Naik ${ }^{2}$, \\ P. Heartwin Amaladhas ${ }^{3}$ and F. Magdaline Eljeeva Emerald ${ }^{3}$
}

${ }^{1}$ College of Dairy Technology, PVNR TVU, Kamareddy, ${ }^{2}$ Department of Dairy Chemistry, ${ }^{3}$ Department of Dairy Engineering, Southern Regional Station, National Dairy Research Institute, Bengaluru, Karnataka, India

*Corresponding author

\section{A B S T R A C T}

Keywords

Green tea,

Catechins, EGCG,

Antioxidative,

Anticarcinogenic,

Anti-inflammatory,

Antimicrobial,

Cardiovascular

disease

Article Info

Accepted:

28 January 2021

Available Online:

10 February 2021
There is an increasing interest in health benefits of foods and facing a large health problems due to rise in the occurrence of lifestyle disorders such as cancer, heart diseases, diabetes and obesity. Therefore, a lot of interest is generated on the dietary substances, especially, the natural ones that enhance the health of the population. Green tea is a 'non-fermented' tea, which is the most popularly consumed beverage in the world. It contains more catechins, than black tea or oolong tea. The health-beneficial effects of green tea are primarily attributed to its polyphenols; around $60-80 \%$ of polyphenols in green tea are catechins, which belong to flavan-3-ols, commonly known as catechins, particularly (-)epigallocatechin-3-gallate. This review depicts the recent findings on the medicinal properties of green tea, focusing on the biological properties of the green tea catechins such as antioxidative, anti-hypertensive, antiobesity, anticarcinogenic, cardioprotective, antibacterial activities and anti-inflammatory. Recommendations are also made to increase the bioavailability of the catechins through nanotechnological delivery mechanisms.

\section{Introduction}

Today, tea is the most regularly consumed beverage in worldwide. There are mainly four types of tea derived from the leaves of Camellia sinensis plant with different processing methods such as green, white, oolong and black tea. The minimal processing, drying is required to produce green and white tea. White tea (non-oxidized) is manufactured from very young leaves and buds, Green tea (non-oxidized) from mature leaves, Oolong tea (partially oxidized) from partially fermented mature leaves, whereas black tea (oxidized) from fully fermented mature leaves (Wanda, 2017). Nearly 2.5 million tons of tea leaves are produced annually all over the world, among that $20 \%$ is green tea (Jayakeerthana, 2016). Green tea has attracted the interest of consumers due to its health benefits against a variety of disorders, ranging from weight loss to cancer. Several reports showed that these non-nutrient bioactive compounds have antioxidant, 
anticancer, antiobesity and other pharmacological and biological functions, thus making them an excellent source for nutraceutical applications. The healthbenefits of green tea are mainly due to their polyphenol content; around $60-80 \%$ of polyphenols are flavan-3-ols, commonly known as catechins. Catechins are the major components of tea; which constitute about $30 \%$ of the dry weight of green tea, and $9 \%$ of black tea (Pradeep, 2017).

\section{Nutritive value of green tea}

Green tea contains complex proteins (15$20 \%$ ), free amino acids (1-4\%) such as glutamic acid, tryptophan, arginine, lysine, glycine, serine, aspartic acid; tyrosine, valine, leucine, threonine and theanine are also present based on dry weight basis. Carbohydrates (5-7\%) in green tea constitute cellulose, fructose, pectins, glucose and sucrose. Minerals and trace elements (5\%) such as calcium, magnesium, iron, copper, zinc, molybdenum, chromium, manganese selenium, sodium, phosphorus, nickel, potassium, fluorine, cobalt, strontium and aluminum are present in green tea on dry weight. Traces of lipids mainly rich in essential fatty acids such as linoleic and $\alpha$ linolenic acids and sterols mainly stigmasterol, vitamins such as $\mathrm{B}, \mathrm{C}$ and $\mathrm{E}$, xanthic bases (caffeine, theophylline), volatile compounds (aldehydes, alcohols, esters, lactones, hydrocarbons) and pigments (chlorophyll, carotenoids) are also present. Many studies have been conducted on tea leaves and its infusions due to its nutraceutical importance. The composition of green tea and green tea infusion is depicted in Table 1. Fresh leaves contain on an average about $3-4 \%$ alkaloids known as methylxanthines, such as caffeine, theobromine, and theophylline (Chaco et al., 2010, Jayakeerthana, 2016). The medicinal properties of green tea are mainly due to the flavonoids. The flavonols (quercetin, kaempferol and rutin), phenolic acids, caffeine, theanine and leucoanthocyanins, account up to $40 \%$ of the dry weight of green tea leaves. Polyphenols are powerful antioxidants and free radical scavengers (Wanda, 2017).

About $80-90 \%$ of the major flavonoids of green tea are various catechins, the four major catechins are mainly (-)-epicatechin (EC), (-)epigallocatechin (EGC), (-)-epicatechin-3gallate (ECG) and (-)-epigallocatechin-3gallate (EGCG). Among these, the most abundant is EGCG ( 59\%) followed by EGC $(\sim 19 \%)$, ECG $(\sim 14 \%)$ and EC $(\sim 6 \%)$ (Anandh and Liu, 2008; Jigisha et al., 2012). Green tea has far higher levels of catechins than black tea. A cup of green tea contains 100-200 mg of EGCG, whereas gallocatechin and catechin are present in trace amounts.

\section{Health benefits of green tea catechins}

The components in green tea undergo metabolic processing such as glucuronidation, methylation and sulfation in the body, which produces some active metabolites. The catechins and their metabolites may be identified in blood plasma, urine, and several tissues (Lambert et al., 2007). However, the bioavailability of catechins is very poor due to various reasons such as poor stability to heat, light, alkaline $\mathrm{pH}$. Recently, the health benefits of green tea and its constituents such as prevention of cancer and cardiovascular diseases and the antioxidative, antiinflammatory, antiarthritic, antibacterial, antiangiogenic, antiviral, neuroprotective and cholesterol-lowering are under investigation.

Molecular mechanisms of green tea catechins

The health benefits of green tea are mainly associated with antioxidant properties and the 
ability of its polyphenolic catechins to scavenge reactive oxygen species (Yang et al., 2004). The difference in the properties are due to their structure, which consists of the phenolic hydroxyl (OH) groups such as ungalloylated catechins on B-ring (EC and EGC) and galloylated catechins (ECG and EGCG) in the B- and D-rings (Figure 1). The presence of 3, 4, 5-trihydroxy B-ring position has been shown important for antioxidant and radical scavenging activity. Rice-Evans et al., (1995) postulated that the green tea catechins were more effective as antioxidants than vitamins $\mathrm{C}$ and $\mathrm{E}$, and the effectiveness of radical scavenger order is shown to be EGCG $>$ EGC $>$ EC $>$ catechin. However, the metal-chelating properties of green tea catechins are also responsible to their antioxidative property (Hider et al., 2001; Kumamoto et al., 2001).

\section{Antioxidant effect}

Nowadays, green tea is one of the most commonly used nutraceuticals due to its antioxidant property. Antioxidant is a substance that inhibits the cellular damage of reactive oxygen species mainly as, singlet oxygen, superoxide, hydroxyl radicals, peroxyl radicals, and peroxynitrite. The disturbance in the balance among antioxidants and ROS results in oxidative stress, which leads to damaging of cells (Chaco et al., 2010). Catechins show the antioxidant activity through various mechanisms as shown in Figure 2: (1) direct mechanism such as scavenging the reactive oxygen species (ROS) and chelating metal ions (2) indirect mechanisms such as inhibiting pro-oxidant enzymes, inducing antioxidant enzymes, and redox sensitive transcription factors (Cabrera et al., 2006). Catechins can stabilize ROS and directly prevents oxidative injury by free radical scavenger (Heim et al., 2002; Nijveldt et al., 2001). The mechanisms of green tea catechins for free radical scavenging activity may involve the electron delocalization, intraand intermolecular hydrogen bonds formation, molecular rearrangement and chelation of metal ions that may be involved in oxidation.

EGCG is considered as one of the most active compound and well known for its strong antioxidant properties, suggesting that the presence of O-trihydroxyl group and 3-gallate esters plays an important role in antioxidant activity, radical scavenging effect and preventing oxidative destruction of many biological compounds (Liao et al., 2001). Consumption of green tea in average limit (16 cups / day) enhanced the plasma and blood antioxidant potential, hence leads to a reduced oxidative damage in macromolecules such as DNA and lipids (Henning et al., 2003).

\section{Anticarcinogenic property}

Currently, cancer is a major source of morbidity and mortality worldwide. Many researchers studied the effect of green tea on cancer therapy (Zaveri, 2006). Mainly EGCG has been extensively used in cancer research. There are several anticarcinogenic mechanisms was attributed to EGCG that may include inhibition of angiogenesis, DNA hypermethylation, NF-kB, telomerase activity, proliferation and metastasis of tumor cells; initiation of tumor suppressor genes and promotion of tumor cell apoptosis as depicted in Figure 3 (Subramani and Natesh, 2013; Butt et al., 2015; Granja et al., 2016).

Epidemiologic studies conducted in Japan reported that cancer onset in, who had consumed 10 cups of green tea every day, was 8.7 years later among women and 3 years later among men, when compared to individuals who had consumed less than 3 cups per day. Cooper et al., (2005) also proposed a possible correlation between high consumption of green tea and low prevalence 
of prostate and breast cancer in Asian countries. However, because of the many variables in lifestyle innate, a definitive relationship between green tea and its cancer prevention effects could not be concluded. Nevertheless, from the above studies, consumption of green tea is beneficial.

Studies in animal models have revealed that green tea and EGCG can inhibit carcinogenesis at all stages, viz. tumour initiation, promotion, malignant conversion and progression (Chung et al., 2003). This multifacrious inhibition of the tumorigenic process is attributed to a combination of antioxidative, antiproliferative and proapoptotic effects. Green tea polyphenols mainly, EGCG have shown to hinder the process of angiogenesis, tumor metastasis and invasion in animal models (Fassina et al., 2004).

\section{Cardiovascular disease}

Cardiovascular disease (CVD) is a class of disorder involving various factors such as inflammation, a disturbance in the balance between the production of ROS (free radicals) and antioxidant defenses, platelet adhesion, and metabolism of lipid. Green tea catechins can show both direct and indirect antioxidant effects on cardiovascular system (Stangl et al., 2007). Hypercholesterolemic rats were treated with green tea and examined for the effect on HMG CoA reductase and other lipid enzymes. They revealed a lowering of serum total cholesterol and elevated HDL levels. The mechanism of action of cardiovascular function was also related to LDL-cholesterol oxidation. The oxidation of LDL cholesterol, associated with a risk for heart disease and atherosclerosis, was inhibited by green tea due to EC and EGCG antioxidant activity. Tea catechins were shown to lower or prevent the deposition of cholesterol accumulation in several tissues, including liver and heart in rats with hypercholesterolemia (Miura et al., 2001).

\section{Antinflammatory effect}

Inflammation is a body response to foreign substances in the human body, leading to damage in the cell tissues. The defense mechanism of anti-inflammatory effect after consumption of green tea catechins showed improvement of production of IL-10 (antiinflammatory cytokine), increase of IL-6 secretion and mediated signaling pathway; reduced production of destructive matrix enzymes such as metalloproteinases via TNF$\alpha$ induced phosphorylation of MAPKs (mitogen-activated protein kinases) and decreased expression of the $\mathrm{CCR}_{2}$ (chemokine receptor) and reduced levels of the proinflammatory cytokines IL-1 and TNF. EGCG is best known for its higher antioxidant activity and also has capacity to decrease the rheumatoid arthritis, inflammation response in the body. EGCG showed strong inhibition of IL- $1 \beta$ inducible nitric oxide synthase (NOS), cyclooxygenase (COX-2) expression and activity in cartilage cell cultures. The overexpressions of NOS and COX-2 are mediated by NF-kB, which can also modulate in the presence of EGCG (Singh et al., 2002).

\section{Antimicrobial property}

Green tea catechins affect the growth of a large number of microorganisms, which include Gram-positive and Gram-negative aerobic bacteria, anaerobic bacteria, viruses and fungi. The antimicrobial mechanisms of green tea catechins are mainly due to the destruction of the bacterial cell membrane, prevention of bacterial fatty acid synthesis and other enzymes such as protein tyrosine kinase, cysteine proteinases, DNA gyrase, ATP synthase and inhibition of efflux pump activity (Wanda, 2017). EGCG and ECG have 
shown marked inhibition of microbial growth at doses more than $25-50 \mu \mathrm{g} / \mathrm{mL}$. The complete inhibition was observed at 100 $\mu \mathrm{g} / \mathrm{mL}$ with EGCG and inhibitory effect of EGCG was higher when compared to ECG (Umegaki et al., 2016). EGCG is also useful in the inhibition of various viruses, such as enterovirus, adenovirus, influenza, hepatitis and herpes virus.

The molecular targets such as NF-kB (Nuclear factor kappa-light-chain-enhancer of activated B cells) and the MAP-kinases pathway are deregulated by the viral infection. EGCG is one of the essential immune response, which helps to combat with the viral infection. The antifungal activity of EGCG was also reported against pathogenic yeasts, such as Candida albicans. However, the mechanism of action was unclear (Mollashahi et al., 2015).

\section{Antiobesity property}

Obesity is characterized as excessive accumulation of fat in the body that may have significant negative impact on overall health and may lead to the development of certain diseases, such as diabetes and arteriosclerosis. EGCG plays an important role, which directly interferes with the lipid digestion by inhibiting the enzyme known as phospholipase $\mathrm{A}_{2}$ and thereby prevents the lipid / cholesterol emulsion interfere in the gut (Granja et al., 2017). The mechanism of EGCG on reduction of obesity and diabetes may also associate with certain pathways, such as energy balance modulation, food intake, endocrine systems, redox status, lipid and carbohydrate metabolism (Chaco et al., 2010). The EGCG is capable of elevating the lipid metabolism, leading to excess burning of calories and ensuing fat loss. It can also interfere with the digestion of starch by inhibiting $\alpha$-amylase. In addition, EGCG ingestion is very useful during a weight loss program because it is strongly related with improvement of circulation, activity of free radical scavenging, and enhancement of mood (Scholay et al., 2012). In vitro studies revealed that green tea extracts containing $25 \%$ of catechins have the ability to inhibit the gastric lipase significantly, and to a lower extent of pancreatic lipase. It is shown that green tea catechins interfere with fat emulsification process, before enzymes acted, and was also essential for lipid intestinal absorption (Chantre and Lairon, 2002). EGCG also exhibited a fatty acid synthase inhibitor activity, reduction on the de novo lipogenesis and secretion of VLDL, having an influence on the increase of adiponectin production, which constitutes activation of AMPK by liver kinase B1 (LKB1) as shown in Figure 4. EGCG showed a potential therapy for the prevention of liver damage in high fat diet associated obesity (Santamarina et al., 2015).

\section{Antidiabetic property}

Epidemiological studies showed that EGCG has a great effect on glucose tolerance and insulin sensitivity. It is associated with the prevention and inhibition of diabetes mellitus through a several effects, such as inhibition of insulin resistance, improvement of insulin secretion, regulation of glucose uptake, increases the glucose tolerance and its role in oxidative stress and inflammation (Chowdhury et al., 2016).

The researchers have also reported that EGCG mimics insulin, which ehnances the tyrosine phosphorylation of insulin receptor and its substrate thereby impede gene expression of the gluconeogenic enzyme PEPCK (phosphoenolpyruvate carboxykinase) enzyme. Currently, the green tea catechins were demonstrated to modify glucose metabolism in experimental models of type II diabetes mellitus. 
Table.1 Approximate composition of green tea and its infusion

\begin{tabular}{|c|c|c|}
\hline \multirow{2}{*}{$\begin{array}{l}\text { Components } \\
\text { Protein }\end{array}$} & Green Tea* & $\begin{array}{l}\text { Green tea Infusion } \uparrow \\
(\%)\end{array}$ \\
\hline & 14.5 & Traces \\
\hline Amino acids & 3.98 & 3.43 \\
\hline Fiber & 25.4 & Nil \\
\hline Carbohydrates & 6.97 & 4.3 \\
\hline Lipids & 6.89 & Traces \\
\hline Pigments & 1.94 & Traces \\
\hline Minerals & 5.2 & 4.45 \\
\hline Phenolic compounds ${ }^{\#}$ & 29.43 & 4.5 \\
\hline Oxidized phenolic compounds ${ }^{\$}$ & Nil & 4.5 \\
\hline
\end{tabular}

* On dry weight basis

$\dagger$ Infusion time: 180 seconds

\# Especially flavonoids

${ }^{\$}$ Especially thearubigins and theaflavins

Fig.1 Structure of major catechins present in green tea

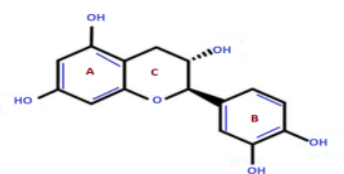

Catechin (C)

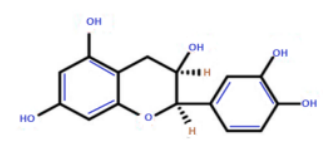

Epicatechin (EC

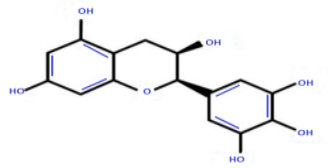

(-)- Epigallocatechin (EGC)

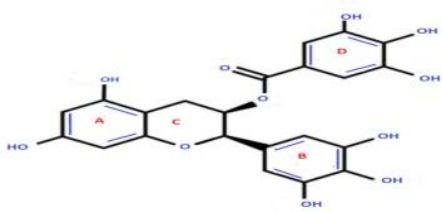

(-)-Epigallocatechin gallate (EGCG)

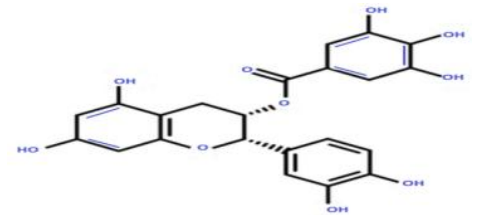

(-)- Epicatechin gallate (ECG)

Fig.2 Antioxidant mechanism of catechins

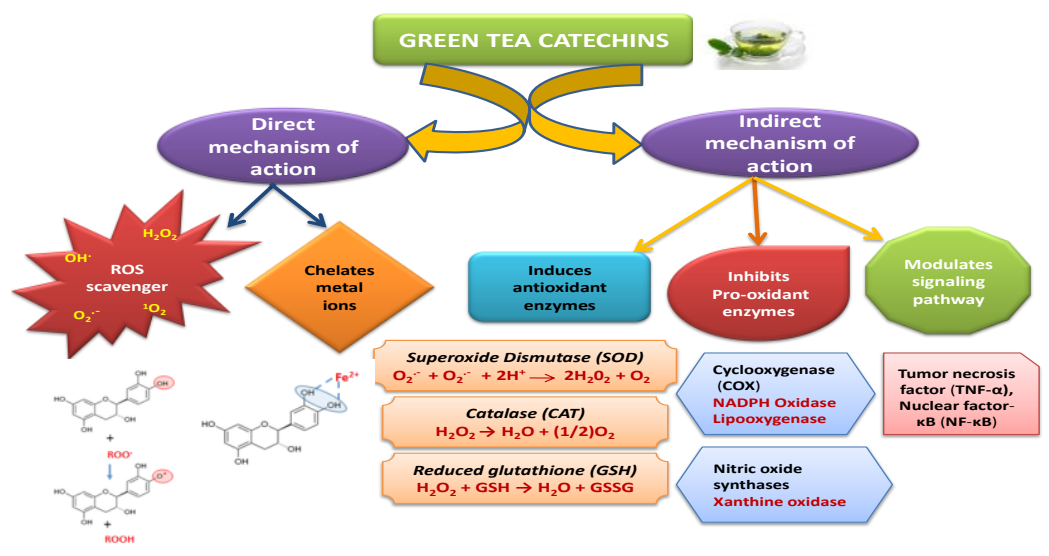


Fig.3 Mechanism of apoptotic cell death by EGCG

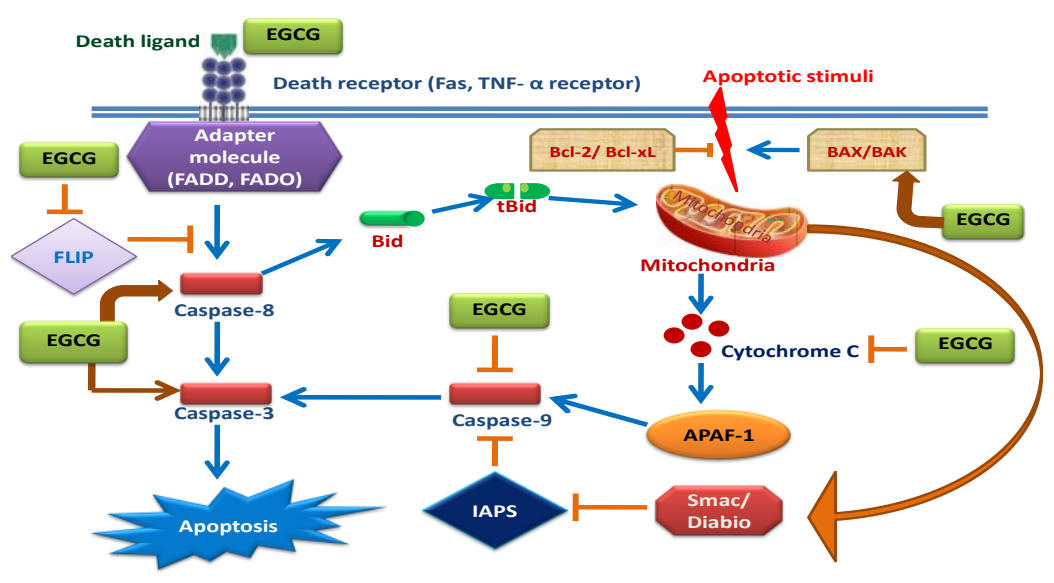

Fig.4 Antiobesity mechanism of EGCG

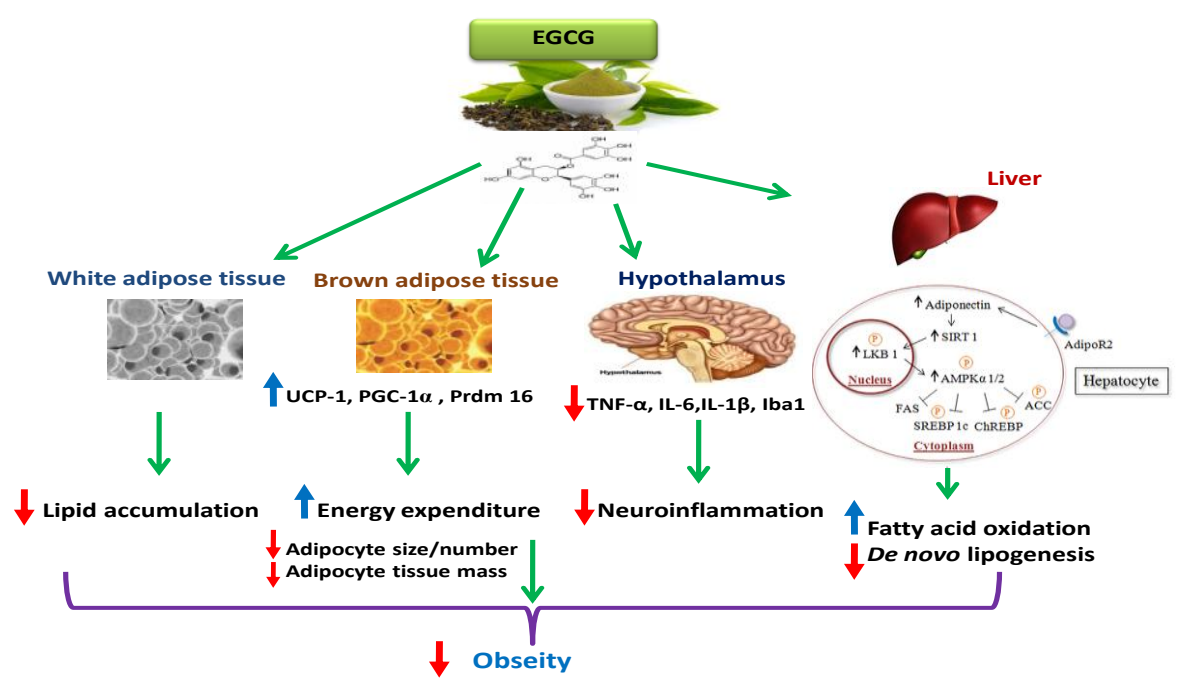

\section{Antihypertensive property}

The observational studies and trials on vasodilation on blood pressure (BP) of green tea and its extracts have been investigated in large quantities for decades. The mechanism associated to antihypertensive effect may be due to the angiotensin peptide hormone that causes vaso- constriction and a subsequent increase in BP, and it is a part of the reninangiotensin system, which is a major target, that lowers the blood pressure. The effect of green tea catechins on hypertension may be related to its inhibition of angiotensin converting enzyme (ACE). Yang et al., (2004) observed that habitual consumption of green tea $120 \mathrm{ml} /$ day for more than a year reduced the risk of high blood pressure significantly. The effect on the ACE activity and antihypertensive is mainly due to the presence of higher amount of O-methylated derivative i.e. (-)-epigallocatechin-3-O-(3-Omethyl) gallate of EGCG (Kurita et al., 2010). 


\section{Other properties}

\section{Dental Caries}

Dental plaque is the major pivotal agent for the initiation of gingivitis. Gingival disease can lead to periodontitis, which if left untreated may finally compromise the entire periodontium. Prevention of dental caries is mainly due to the EGCG, which will bind and inhibits salivary and bacterial amylases, especially $\alpha$-amylase. It also stops the acid generation from carbohydrates by inhibiting the transcription and function of lactate dehydrogenase (LDH). Oral bacteria such as Streptococcus mutans, plays a major role in promoting the tooth decay, which forms a biofilm on the surface of teeth. EGCG may inhibit or remove the bacteria from teeth, thereby decreases the formation of biofilm and inhibits the bacteria to develop an acid environment. EGCG also hinders the hydrogen binding and hydrophobic interactions of collagenolytic bacteria. Green tea consumption regularly increases the oral peroxidase activity (Kato et al., 2009, Hara et al., 2012, Narotzki et al., 2013).

\section{Effect on skin}

In vitro and in vivo animal and human studies demonstrated that green tea catechins are photoprotective in nature. The clinical trials in humans showed the inhibition of solar ultraviolet B light induced skin disorders such as photoaging, melanoma and non melanoma skin cancers (Katiyar, 2003).

\section{Limitations of catechins}

Although the catechins reported to have therapeutic effects, their applicability to humans is limited. Catechins are very sensitive and unstable to oxidising enzymes, heat, and high $\mathrm{pH}$. They have limitations such as poor bioavailability and stability. Its consumption is not preferred by many due to its odd taste.

\section{Bioavailability of catechins}

Catechins readily undergo a number of metabolic processes such as glucuronidation, methylation, and sulfation in the body, by intestinal microflora and enzymes, are poorly bioavailable (Pradeep et al., 2017, Qiao et al., 2014). The catechins and its metabolites may be identified in blood plasma, urine, and several tissues (Lambert et al., 2007). In humans, the concentration of catechins in plasma primarily increased from 2 to 4 hours. However, the bioavailability of catechins is relatively lower; the plasma catechin (EGCG and EGC) concentration was only 0.2 to $2 \%$ when ingested in healthy people, which affects the biological activity within the target tissues (Cabrera et al., 2006). Therefore, bioavailability of catechin represents a big challenge. Generally, catechins are easily metabolized by enzymes and microbes and this group of compounds have short plasma half-lives (2-3 h). The half-lives of green tea catechins are 2-4 $\mathrm{h}$ and their absorption and removal are rapid in humans. The peak times are in the range of 1 and $3 \mathrm{~h}$ with low peak plasma concentrations in the $\mu \mathrm{M}$ range $(\mathrm{Fu}$ et al., 2008). Another study emphasized that the bioavailability of the green tea catechins was shown to be low, resulting in systemic levels in humans 5 to 50 times less than concentrations showed biological activities in in vitro systems. Results from other researchers also confirmed the bioavailability of catechins was lower than 5\% (Catterall et al., 2003).

\section{Stability of catechins}

Catechins are sensitive to several environmental factors such as heat and light, and readily undergo degradation in oxidation with a consequent loss in activity. A number 
of studies have shown the unstability of catechins compounds. One study focusing on the $\mathrm{pH}$ stability of catechin isomers from green tea found that they were extremely unstable and degraded almost completely in a few minutes in the alkaline solution $(\mathrm{pH}>8)$, whereas in the acidic solution $(\mathrm{pH}<4)$ they were very stable at least for $18 \mathrm{~h}$ (Pradeep et $a l ., 2017)$. The stability of green tea catechins in sodium phosphate buffer with a $\mathrm{pH}$ ranging between 5.0 and 7.4 showed a $\mathrm{pH}$-dependent trend. Among the various catechin isomers, EGCG was quite unstable shown almost complete degradation when incubated for $3 \mathrm{~h}$ at $\mathrm{pH}$ 7.4. Other researchers also reported that green tea catechins were more vulnerable to degradation caused by elevation of temperature, $\mathrm{pH}$ and chelating metal ions (Sang et al., 2005). The unstability is part of the reason for the poor bioavailability and also presents as an issue in the manufacture process.

\section{Strategies to overcome limitations}

Several novel and promising nanotechnological methods have been employed to improve bioavailability of catechins such as nanoemulsion, niosomes etc. Nanoencapsulation of catechins represents a viable and efficient approach to increase their physical stability under GI conditions, protecting them from interacting with other components of digestion and premature degradation in the body. It helps in increased bioactivity due to the nanosize. Biodegradable nanoparticles of $100 \mathrm{~nm}$ size showed 15- to 250-fold higher uptake efficiency as compared to larger sized microparticles $(>500 \mathrm{~nm})$. Studies on nanoencapsulation revealed that EGCG stability was significantly increased, enhanced bioavailability and improved its sustained release in controlled manner, which may partially increased cellular uptake of EGCG. Therefore nanoencapsulation of catechin helps to increase their bioavailability and can preferably be used as delivery mechanism for fortification in foods.

In conclusion nowadays, green tea extracts are considered as one of the most promising nutritional therapy for several diseases and consequently, it is being studied enormously worldwide. A number of epidemiological scientific studies have confirmed the well being of green tea catechins. In this review we covered physiological benefits of green tea catechins such as antioxidant, antimutagenic, antidiabetic, anti-inflammatory, antimicrobial and antiviral, and above all, cancer-preventive properties. Though all the evidence from research on green tea is very positive, future studies are necessary to understand its contributions and impact on human health, and advice its regular consumption in western diets, in which green tea consumption is limited. Nanoencapsulation of green tea catechins is a promising tool for increasing their bioavailability and effective delivery.

\section{Conflicts of Interest}

The authors and co- authors declare no conflicts of interest.

\section{References}

Anandh, B. P. V., and Liu, D. 2008. Green Tea Catechins and Cardiovascular Health: An Update. Current Medicinal Chemistry, 15, 1840-1850.

Butt, M. S., Ahmad, R. S., Sultan, M. T., Qayyum, M. M., and Naz, A. 2015. Green tea and anticancer perspectives: Updates from last decade. Critical Reviews in Food Science and Nutrition, 55, 792-805.

Cabrera, C., Artacho, R., and Gimenez, R. 2006. Beneficial effects of green teaA review. Journal of the American College of Nutrition. 25(2), 79-99. 
Catterall, F., King, L., Clifford, M., and Ioannides, C. 2003. Bioavailability of dietary doses of $3 \mathrm{H}$-labelled tea antioxidants (+)-catechin and (-)epicatechin in rat. Xenobiotica, 33(7), 743-753.

Chaco, S. M., Priya, T. T., Ramadasan, K., and Nishigaki, I. 2010. Beneficial effects of green tea: A literature review. Chinese Medical, 5, 13.

Chantre, P., and Lairon D. 2002. Recent findings of green tea extract AR25 (exolise) and its activity for the treatment of obesity. Phytomedicine, 9, 3-8.

Chowdhury, A., Sarkar, J., Chakraborti, T., Pramanik, P. K., and Chakraborti, S. 2016. Protective role of epigallocatechin-3- gallate in health and disease: a perspective. Biomed. and Pharmacotherapy, 78, 50-59.

Chung, F.L., Schwartz, J., Herzog, C.R., Yang, Y.M., 2003. Tea and cancer prevention: studies in animals and humans. Journal of Nutrition, 133 (10), 3268S-3274S.

Cooper, R., Morre, J., Dorothy, M., and Morree. 2005. Medicinal benefits of green tea: Part II. Review of anticancer properties, Journal of Alternative and Complementary Medicine, 11(4), 639-652

Fassina, G., Vene, R., Morini, M., Minghelli, S., Benelli, R., Noonan, D.M., Albini, A. 2004. Mechanisms of inhibition of tumor angiogenesis and vascular tumor growth by epigallocatechin-3gallate. Clinical Cancer Research, 10 (14), 4865-4873.

Fu, T., Liang. J., Han G., Lv. L. and Li N. 2008. Simultaneous determination of the major active components of tea polyphenols in rat plasma by a simple and specific HPLC assay. Journal of Chromatography B, 875(2), 363-367.

Granja, A., Frias, Iuri., Neves, A. R.,
Pinheiro, Marina and Reis, Salette. 2017. Therapeutic Potential of Epigallocatechin Gallate. Nanodelivery Systems. BioMed Research International, https://doi.org/10.1155/2017/5813793

Granja, A., Pinheiro, M., and Reis, S. 2016. Epigallocatechin gallate nanodelivery systems for cancer therapy. Nutrients, 8, E307.

Hara, K., Ohara, M., Hayashi, I., Hino, T., Nishimura, R., Iwasaki, Y., Ogawa, T., Ohyama, Y., Sugiyama, M., and Amano, H. 2012. The green tea polyphenol (-)-epigallocatechin gallate precipitates salivary proteins including alpha-amylase: Biochemical implications for oral health. European Journal of Oral Sciences, 120, 132139.

Heim, K. E., Tagliaferro, A. R., Bobilya, D. J. 2002. Flavanoids antioxidants: chemistry, metabolism and structureactivity relationships. Journal of Nutritional Biochemistry, 13, 572.

Henning, S, M., Fajardo-Lira C., Lee HW, Youssefian AA, Go VLW., and Heber D. 2003. Catechin contents of 18 teas and green tea extract supplement correlates with the antioxidant capacity. Nutrition and Cancer, 45, 226-235.

Hider, R. C., Liu, Z. D., and Khodr, H. H. 2001. Metal chelation of polyphenols. Methods in Enzymology , 335, 190203.

Jayakeerthana, S. 2016. Benefits of Green Tea: A Review. Journal of Pharmaceutical Sciences and Research, 8(10), 1184-1187

Jigisha, A., Nishant, R., Navin, K., Pankaj, G. 2012. Green tea: A magical herb with miraculous outcomes. International Research Journal of Pharmacy, 3, 139-148.

Katiyar, S. K. 2003. Skin photoprotection by 
green tea: antioxidant and immunomodulatory effects. Bentham science publishers Current Drug Targets - Immune, Endocrine and Metabolic Disorders, 3(3), 234-242.

Kato, M.T., Magalháes, A.C., Rios, D., Hannas, A. R., Attin, T., and Buzalaf, M.A. 2009. Protective effect of green tea on dentin erosion and abrasion. Journal of Applied Oral Science, 17, 560-564.

Kumamoto, M., Sonda, T., Nagayama, K., and Tabata, M., 2001. Effects of $\mathrm{pH}$ and metal ions on antioxidative activities of catechins. Bioscience, Biotechnology, and Biochemistry, 65 (1), 126-132.

Kurita, I., Maeda-Yamamoto, M., Tachibana, H., and Ka- mei, M. 2010. Antihypertensive effect of Benifuuki tea containing O-methylated EGCG. Journal of Agricultural and Food Chemistry. 58, 1903-1908.

Lambert, J. D., Sang, S., and Yang, C.S. 2007. Biotransformation of green tea polyphenols and the biological activities of those metabolites. Molecular Pharmacology, 4, 819-825.

Liao, S., Kao, Y. H., and Hiipakka, R. A. 2001. Green tea: Biochemical and biological basis for health benefits. Vitamins and hormones, 62, 1-94.

Miura, Y., Chiba, T., Tomita, I., Koizumi, H., Miura, S., Umegaki, K., Hara, Y., Ikeda, M., and Tomita, T. 2001, Tea Catechins Prevent the Development of Atherosclerosis in Apoprotein EDeficient Mice. Journal of Nutrition, 131(1), 27-32,

Mollashahi, N. F.; Bokaeian, M., Mollashahi, L, F and Afrougheh, A. 2015. Antifungal efficacy of green tea extract against candida albicans biofilm on tooth substrate. Journal of Dentistry, 12(8), 592.

Narotzki, B., Levy, Y., Aizenbud, D.,
Reznick, A.Z. 2013. Green tea and its major polyphenol EGCG increase the activity of oral peroxidases. Advances in Experimental Medicine and Biology, 756, 99-104.

Nijveldt, R. J., van Nood, E., van Hoorn, D. E., Boelens, P. G., Van Norren, K.; Van Leeuwen, P. 2001. Flavanoids : a review of probable mechanisms of action and potential applications. American Journal of Clinical Nutrition, 74, 418.

Pradeep, P., Chulkyoon, M., Sanghoon, K., Liang, J., Neeraja, R., 2017. Nanotechnological approaches to enhance the bioavailability and therapeutic efficacy of green tea polyphenols. Journal. of Functional Foods. 34, 139-151.

Qiao, J., Kong, X., Kong, A., and Han, M. 2014. Pharmacokinetics and Biotransformation of Tea Polyphenols. Current Drug Metabolism, 15(1), 3036.

Rice-Evans, C.A., Miller, N.J., Bolwell, P.G., Bramley, P.M., and Pridham, J.B., 1995. The relative antioxidant activities of plant-derived polyphenolic flavonoids. Free Radical Research, 22 (4), 375-383.

Sang, S., Lee, M.J., Hou, Z., Ho, C.-T. and Yang, C. S. 2005. Stability of tea polyphenol (-)-epigallocatechin-3gallate and formation of dimers and epimers under common experimental conditions. Journal of Agriculture and Food Chemistry, 53(24), 9478-9484.

Santamarina, A. B., Juliana, L., Oliveira, Fernanda P., Silva, June Carnier, Lais V., Mennitti, Aline A. et al., 2015. Green Tea Extract Rich in Epigallocatechin3-Gallate Prevents Fatty Liver by AMPK Activation via LKB1 in Mice Fed a High-Fat Diet. Plos One, 11(4), 1-18.

Scholey, A., Downey, L. A., Ciorciari, J. et 
al., 2012. Acute neurocognitive effects of epigallocatechin gallate (EGCG). Appetite, 58(2), 767-770.

Singh, R., Ahmed, S. Islam, N. Goldberg, V. M. and Haqqi, T. M. 2002. Epigallocatechin-3-gallate inhibits interleukin- $1 \beta$ - induced expression of nitric oxide synthase and production of nitric oxide in human chondrocytes: Suppression of nuclear factor $\kappa \mathrm{B}$ activation by degradation of the inhibitor of nuclear factor $\kappa \mathrm{B}$," Arthritis and Rheumatism. 46(8). 2079- 2086.

Stangl, V., Dreger, H., Stangl, K., and Lorenz, M. 2007. Review molecular targets of tea polyphenols in the cardiovascular system. Cardiovascular Research, 73, 348.

Subramani, C., and Natesh, R.K. 2013, Molecular mechanisms and biological implications of green tea polyphenol, (-)-epigallocatechin-3-gallate.
International Journal of Pharma Bio science and Technology, 1, 54-63.

Umegaki, K., Seine, Y., Sato, Y., Chiba, T. and Sonoda, M. 2016. Effect of Tea Catechins on folate analysis in green tea by microbiological assay. Journal of Nutritional Science and Vitaminology, 62, 134-138.

Wanda, C. R. 2017. An Update on the Health Benefits of Green Tea. Beverages, 3(6), 1-14.

Yang YC, Lu FH, Wu JS, Wu CH, Chang C,J. 2004. The protective effect of habitual tea consumption on hypertension. Archives of internal medicine, 164, 1534-1540.

Zaveri, N. T. 2006. Green tea and its polyphenolic catechins: Medicinal uses in cancer and noncancer application. Life Sciences, 78, 20732080.

\section{How to cite this article:}

Swarnalatha, G., B. Surendra Nath, N. Laxmana Naik, P. Heartwin Amaladhas and Magdaline Eljeeva Emerald, F. 2021. Therapeutic Potential of Green Tea Catechins - A Magical Herb. Int.J.Curr.Microbiol.App.Sci. 10(02): 3560-3571. doi: https://doi.org/10.20546/ijcmas.2021.1002.392 